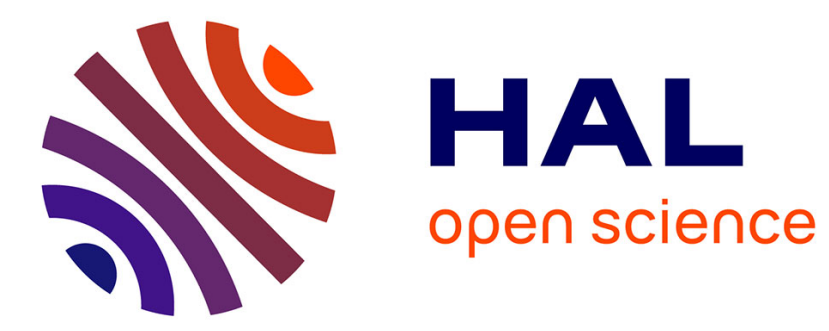

\title{
The influence of semantic processing and response latency on the SNARC effect
}

\author{
Daniele Didino, Christina Breil, André Knops
}

\section{To cite this version:}

Daniele Didino, Christina Breil, André Knops. The influence of semantic processing and response latency on the SNARC effect. Acta Psychologica, 2019, 196, pp.75-86. 10.1016/j.actpsy.2019.04.008 . hal-02454395

\section{HAL Id: hal-02454395 \\ https://hal.science/hal-02454395}

Submitted on 24 Jan 2020

HAL is a multi-disciplinary open access archive for the deposit and dissemination of scientific research documents, whether they are published or not. The documents may come from teaching and research institutions in France or abroad, or from public or private research centers.
L'archive ouverte pluridisciplinaire HAL, est destinée au dépôt et à la diffusion de documents scientifiques de niveau recherche, publiés ou non, émanant des établissements d'enseignement et de recherche français ou étrangers, des laboratoires publics ou privés. 
Title: The influence of semantic processing and response latency on the SNARC effect

2

3

4

5

6

7

8

9

10

11

Daniele Didino ${ }^{\mathrm{a}^{\star}}$, Christina Breil ${ }^{\mathrm{a}}$, André Knops $^{\mathrm{b}, \mathrm{c}}$

a. Department of Psychology, Faculty of Life Sciences, Humboldt-Universität zu Berlin, Berlin, Germany.

b. CNRS UMR 8240, Laboratory for the Psychology of Child Development and Education, Paris, France.

c. University Paris Descartes, Sorbonne Paris Cité, Paris, France.

* Corresponding author:

Daniele Didino

Department of Psychology, Humboldt-Universität zu Berlin, Rudower Chaussee 18, 12489

Berlin, Germany. E-mail: daniele.didino@gmail.com

Competing interests: The authors declare that they have no conflict of interest. 


\section{INTRODUCTION}

In their seminal paper, Dehaene, Bossini and Giraux (1993) asked participants to classify the parity of numbers by pressing a left- or right-sided key (i.e., parity judgement task). They discovered that relative small numbers elicit faster left-sided responses and large numbers faster right-sided responses. Since then, this Spatial-Numerical Association of Response Codes effect (SNARC; for reviews see Fisher \& Shaki, 2014; van Dijck et al., 2015; Wood et al., 2008) has been replicated multiple times.

Originally, the SNARC effect has been explained within the framework of the mental number line (MNL) account (Dehaene et al., 1993). The MNL is a semantic long-term memory representation in which numbers are coded on a unidimensional conceptual space, horizontally organized in ascending order from left to right: small numbers are encoded on the left side and large numbers on the right side (Hubbard et al., 2005). This spatial organization is thought to emerge from cultural practices such as writing/reading direction (Göbel, 2015; Göbel, Maier, \& Shaki, 2015; Göbel, Shaki, \& Fischer, 2011; for a right-to-left spatial orientation of the MNL in non-Western cultures see Shaki, Fischer, \& Göbel, 2012; Shaki, Fischer, \& Petrusic, 2009). According to the MNL account, the SNARC effect emerges from an isomorphism (i.e., a direct mapping) between the position of a number on this semantic representation and the left-right coordinates of the external response locations. Therefore, a congruent mapping between conceptual (e.g., small numbers) and external space (e.g., leftsided responses) generates faster response latencies compared to an incongruent mapping (e.g., small numbers and right-sided responses), and vice versa for large numbers.

In the majority of the tasks used to investigate the SNARC effect (e.g., parity judgement task) the magnitude of the number is irrelevant for the decision process (for a list of tasks see Wood et al., 2008). Therefore, the MNL account proposes that the SNARC effect emerges from the interference produced by an automatic activation of the semantic representation of numbers (Dehaene et al., 1993; Nuerk, Wood, \& Willmes, 2005). An important question pertains to whether and how the amount of semantic processing (i.e., processing of abstract properties of numbers such as magnitude or parity) required by the task modulates the level of interference generated by the MNL. In line with the MNL account, one would expect that deeper semantic processing of a number leads to a stronger activation of the MNL and in turn more interference (i.e., a stronger SNARC effect) is expected. Wood and colleagues (2008) evaluated this hypothesis in a meta-analysis which was consistent with the MNL account. Tasks requiring deeper semantic processing (e.g., parity judgement and magnitude classification) were associated with a stronger SNARC effect compared to tasks asking to discriminate non-semantic features of the numbers (e.g., color judgement) (see table 1 and 
figure 2 in Wood et al., 2008). However, to the best of our knowledge, no study tested this hypothesis in a within-participant design.

The assumption of a direct mapping between the position of a number on the MNL and response location coordinates has been challenged by the dual route model (Gevers et al., 2006, 2010; Santens \& Gevers, 2008) and the working memory (WM) account (Abrahamse, van Dijck, \& Fias, 2016; Fias \& van Dijck, 2016; van Dijck \& Fias, 2011; van Dijck, Gevers, \& Fias, 2009; van Dijck et al., 2012, 2014). Neither of these accounts predicts that the strength of the SNARC effect is affected by the level of semantic processing required by a task.

The dual route model (Gevers et al., 2006, 2010; Santens \& Gevers, 2008; see also the polarity correspondence account for a similar idea not restricted to number processing, Proctor and Cho, 2006) introduces an intermediate level of processing, between the MNL and the response stage, that codes numbers into binary categories such as magnitude (i.e., small vs. large), parity (i.e., odd vs. even) or other dichotomous features of the stimulus. As the name suggests, this model includes two routes of parallel information processing. The unconditional route codes numbers based on their magnitude and is automatically activated regardless of the task requirements. This route activates the long-term preexisting links between magnitude and space coordinates, that is the associations small-left and large-right. The conditional route codes numbers into a binary category based on task-specific requirements. It activates short-term links between numbers and an arbitrary mapping based on task requirements, such as small-right and large-left (for magnitude) or odd-right and even-left (for parity). The SNARC effect emerges from the congruence, or lack of it, between the response sides activated by the two routes. On the one hand, the conditional route activates a response side based on task-specific mapping. On the other hand, the unconditional route can cooperate or compete to activate the same or the opposite response side, respectively. If the task-related mapping is consistent with the long-term number-space links the two routes cooperate, otherwise they converge on opposite spatial response codes. The response selection process takes longer and response latency is slower when the routes diverge and this generates the SNARC effect. Since both routes are always simultaneously activated independent of the task, the level of semantic processing required by the task should not influence the SNARC effect. According to the dual-route model, however, the strength of the SNARC effect is influenced by the duration of the number processing (Gevers et al., 2006). Namely, the strength of the SNARC effect would increase along with response latency, because longer latency provides the unconditional route with more time to interfere with response selection. Finally, the model also predicts that in magnitude comparison or classification tasks the SNARC effect takes a step-like shape (Gevers et al., 2006), indicating a categorical decision. In tasks that do not require to evaluate the magnitude of a number (e.g., parity judgement task), the SNARC effect is stronger for the extremes of the numerical interval presented in the experiment and thus it 
takes a linear shape (for a review see Wood et al., 2008; but see Schroeder, Nuerk \& Plewnia, 2017c). On the other hand, in a magnitude classification task, where the numbers have to be compared to a fixed standard (e.g., the numbers 1 to 9 being classified as smaller or larger than 5), the SNARC effect interacts with the distance effect (i.e., the time required to compared two numbers is inversely proportional to their numerical distance; Moyer \& Landauer, 1967; Verguts, Fias, \& Stevens, 2005). Therefore, the numbers closer to the standard require more time to be classified and thus the unconditional route has more time to interfere with the response selection. This increases the strength of the SNARC effect for numbers in the center of the interval and thus the effect acquires a categorical rather than linear shape (see figures 2 and 3 in Gevers et al., 2006).

Both the MNL account and the dual-route model assume that the association between numbers and space coordinates are an intrinsic property of the long-term memory representation of number. The working memory (WM) account instead assumes that no spatial information is co-represented together with numbers and that the long-term representation only includes serial order information (Abrahamse, van Dijck, \& Fias, 2016; Fias \& van Dijck, 2016; van Dijck \& Fias, 2011; van Dijck, Gevers, \& Fias, 2009; van Dijck et al., 2012, 2014). The number-space association would emerge from a temporary binding of numbers to a spatially oriented template in working memory. Therefore, the WM account proposes that the SNARC effect is produced by this temporary left-to-right orientation that numbers (considered as verbal items) take only after being transferred to a spatial working memory template. Evidence in favor of the coexistence of long-term space-number associations (i.e., spatially oriented MNL) and temporary associations between space and order position in working memory (Ginsburg \& Gevers, 2015; Huber et al., 2016; Schroeder, Nuerk \& Plewnia, 2017c) can be reconciled with the WM account working assuming that multiple serially ordered sequences of numbers can be simultaneously activated in working memory (Abrahamse, van Dijck, \& Fias, 2016). Reverse SNARC-like effect for ordinal non-numerical sequences (e.g., weekdays) under transcranial direct current stimulation (Schroeder, Nuerk \& Plewnia, 2017a) also challenges the WM account, but mixed evidence emerged for such effect (Schroeder, Nuerk \& Plewnia, 2017b). Since the spatial orientation is not a feature of the long-term memory representation but rather emerges from short-term association in working memory, the level of processing required by the task should not affect the strength of the SNARC effect. Therefore, the WM account also predicts no influence of the amount of semantic processing on the strength of the SNARC effect.

If one strictly defines "semantic processing" as reflecting the access to and the manipulation of numerical magnitude, the fact that the SNARC effect was originally discovered in a parity judgement task (Dehaene et al., 1993), which does not require magnitude processing, already suggests that the amount of semantic processing required by the task does not influence the 
strength of this effect. Wood and colleagues' review (2008), which showed that the size of the SNARC effect in magnitude classification and parity judgement tasks is comparable, also provides evidence for a lack of influence of semantic processing. However, although unrelated with numerical magnitude, parity is actually an intrinsic property of both non-symbolic numerosities (reflecting the possibility to divide a set of objects in two subsets with equal cardinality) and symbolic numbers (reflecting the property of being divisible by 2 with no remainder, in the natural numbers set). Therefore, a parity judgement task requires explicitly to process an abstract semantic (unrelated to magnitude) property of the number. Since in both parity judgement and magnitude classification tasks participants are asked to evaluate an intrinsic property of the number, it is plausible to assume that these two tasks do not allow to clearly disentangle the depth of the semantic processing and thus to measure properly its influence on the SNARC effect. Therefore, to investigate the effect of semantic processing on the SNARC effect, it is more convenient to compare tasks requiring deeper semantic processing (e.g., parity judgement and magnitude classification) with tasks in which response selection is not related to semantic features of the numbers (e.g., color judgement and phoneme detection, see description below). In fact, although to some extent the mere presentation of a number may suffice to automatically activate its semantic representation (Dehaene et al., 1998; Koechlin et al., 1999; Naccache \& Dehaene, 2001), the automatic processing of semantic information is not compulsory (Kunde, Kiesel \& Hoffmann, 2003; Maxfield, 1997). Therefore, we assumed that tasks requiring to assess non-numerical properties of the stimulus (e.g., color judgement and phoneme detection) will evoke a weaker activation of the semantic representation compared to tasks that directly require the discrimination of magnitude or parity.

The present study aimed to evaluate whether and how the amount semantic processing required by a task affects the strength of the SNARC effect, as predicted by the MNL approach. Participants performed two tasks requiring semantic number processing (magnitude classification and parity judgement) and two tasks requiring the discrimination of non-semantic features of the numbers (color judgement and phoneme detection). According to the MNL account, a stronger SNARC effect is expected for the magnitude classification task and parity judgement task compared to the other two tasks. According to the dual-route model, the SNARC effect should be modulated by response latency rather than by the amount of semantic processing. Moreover, this model predicts a categorical shape of the SNARC effect for the magnitude classification task and a linear shape for the other tasks. Finally, similar to the dualroute model, the WM account does not predict that tasks requiring deeper semantic processing (magnitude classification and parity judgement) generate a stronger SNARC effect compared to those based on more peripheral features of the numbers (color judgement or phoneme detection). 
Further predictions can also be made on the relationship between the SNARC effects evoked in the different tasks ${ }^{1}[\mathrm{DD} 1]$. The MNL account assumes the same origin for the SNARC effect regardless of the task. In fact, independently of the task requirements, a common semantic representation is automatically (or deliberately) activated and interferes with the response selection. Given the common origin of this interference, in addition to the expected relationship between SNARC effect and amount of semantic processing, the MNL account also predicts strong correlations between the strength of this effect in the different tasks. The dualroute model, on the other hand, assumes that the SNARC effect originates from the competition/cooperation between two routes of parallel information processing. In other words, in different tasks the unconditional route competes or cooperates with distinct conditional routes, which reflect short-term task-specific mappings. Therefore, the dual-route model does not expressly entail a correlation between the SNARC effects of the different tasks. The WM account assumes that the SNARC effect emerges from the temporary spatial orientation that numbers acquire after being loaded in a working memory template. The binding of numbers to this spatial template is both context- and task-specific. Therefore, similar to the dual-route model, the WM account also does not expressly entail a correlation between the SNARC effects evoked by the different tasks.

\section{METHOD}

\subsection{Participants}

Thirty-two German-speaking participants took part in the study (22 female; mean age (SD) $=26.7$ (4.4), range $=18-35$ ). All participants had normal or corrected-to-normal vision and gave informed consent to participate for course credits or $8 €$. All participants were right-handed and had no experience with right-to-left writing/reading languages. The study was approved by the Ethics committee at the Department of Psychology of Humboldt-Universität zu Berlin (Nr. 2017-29).

\subsection{Materials, tasks and design}

The same set of numbers (Arabic digits ranging from 1 to 9, excluding 5) was used in all tasks. Each participant performed four tasks. In the magnitude classification task, participants were asked to decide if the presented number was smaller or larger than the fixed standard 5. In the parity judgement task, were asked to judge if the number was even or odd. In the color judgement task, participants classified the color of the font: numbers 2, 3, 6 and 8 were always printed in red (RGB: 255, 0, 0) and numbers 1, 4, 7 and 9 always in green (RGB: 0, 255, 0). In

\footnotetext{
${ }^{1}$ We would like to thank Wim Gevers for pointing out this idea.
} 
the phoneme detection task, participants decided whether or not any of the phonemes $|s|,|z|$ and /ts/ was included in the German number word corresponding to the presented Arabic digit (for a similar task see Fias, 2001; Fias et al., 1996). This rule was selected to create an arbitrary mapping (i.e., not related to semantic features) between numbers and response sides. Therefore, numbers were divided into two groups based on the presence ( $1=/$ aInns $/, 2=/$ tsvaI $/$,

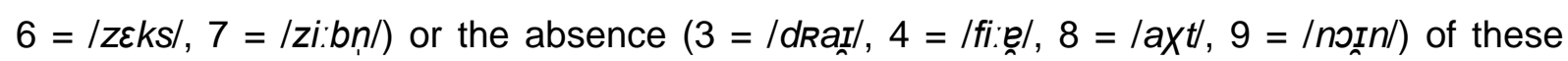
phonemes. Participants were explicitly instructed to base their decision on the phonological feature and ignore the orthography of the word.

Four task sequences were created using the following rules: (1) each task must be presented in each position of the sequence only once; (2) each task can follow the other tasks only once. The four sequences were: Magnitude-Color-Parity-Phonology; ParityMagnitude-Phonology-Color; Phonology-Parity-Color-Magnitude; Color-PhonologyMagnitude-Parity. Each sequence was administered to 8 participants. Each task included two blocks, in which the response mapping was reversed (Table 1). Fifteen participants started all tasks with the response mapping $A$ and 17 participants with the response mapping $B$ (the sample size in the two groups is not equal because of a technical problem). In total, participants performed 8 blocks ( 4 tasks $\times 2$ response mappings). Following the recommendations of Cipora and Wood (2017), in each block each number was repeated 20 times. Numbers were pseudo-randomly presented with the constraint that the same digit could not be presented on two consecutive trials. Sixteen practice trials preceded each block (each number presented twice in a randomized order) to familiarize with the block-specific response mapping and feedback was provided (no feedback was presented during the test blocks).

Table 1. The two response mappings used in the tasks. Response mappings $A$ and $B$ were presented in two different blocks in counterbalanced order across participants.

\begin{tabular}{|l|l|lllllllll|}
\cline { 2 - 11 } \multicolumn{1}{c|}{} & $\begin{array}{l}\text { Response } \\
\text { mapping }\end{array}$ & \multicolumn{3}{c|}{ Left key } & \multicolumn{4}{c|}{ Right key } \\
\hline $\begin{array}{l}\text { Magnitude } \\
\text { task }\end{array}$ & $\mathrm{A}$ & 1 & 2 & 3 & 4 & 6 & 7 & 8 & 9 \\
\cline { 2 - 11 } & $\mathrm{B}$ & 6 & 7 & 8 & 9 & 1 & 2 & 3 & 4 \\
\hline $\begin{array}{l}\text { Parity } \\
\text { task }\end{array}$ & $\mathrm{A}$ & 1 & 3 & 7 & 9 & 2 & 4 & 6 & 8 \\
\hline \multirow{2}{*}{$\begin{array}{l}\text { Phonological } \\
\text { task }\end{array}$} & $\mathrm{B}$ & 2 & 4 & 6 & 8 & 1 & 3 & 7 & 9 \\
\hline \multirow{2}{*}{$\begin{array}{l}\text { Color } \\
\text { task }\end{array}$} & $\mathrm{B}$ & 1 & 2 & 6 & 7 & 3 & 4 & 8 & 9 \\
\cline { 2 - 11 } & $\mathrm{B}$ & 3 & 4 & 8 & 9 & 1 & 2 & 6 & 7 \\
\cline { 2 - 10 } & $\mathrm{B}$ & 2 & 3 & 6 & 8 & 1 & 4 & 7 & 9 \\
\hline
\end{tabular}

\subsection{Procedure}

The same procedure was used in all tasks. Stimulus presentation and response collection were implemented in Matlab, using the Psychophysics Toolbox (Brainard, 1997; Pelli, 1997). Stimuli were presented in the center of the monitor and were $22 \mathrm{~mm}$ high and $15 \mathrm{~mm}$ wide. Participants sat at approximately $50 \mathrm{~cm}$ from the monitor (visual angle: $2.5^{\circ} \times 1.7^{\circ}$ ). 
Each trial started with a fixation mark (\#) presented for $600 \mathrm{~ms}$, followed by an Arabic number, which remained on the screen until the response or for $1300 \mathrm{~ms}$. Participants were instructed to press a key on the left ("left-control" with the left-hand) or right ("enter" on the numpad with the right-hand) side of the keyboard according to the block-specific response mapping (Table 1). The two keys were approximately $40 \mathrm{~cm}$ apart. Following the offset of the presented number, the next trial began after an intertrial-interval of 500 ms consisting of a black screen. All stimuli were printed in white against a black background, except for numbers in the color judgement which could be red (RGB: 255,0,0; numbers 2, 3, 6 and 8) or green (RGB: 0. 255, 0; 1, 4, 7, 9).

Since participants had to perform eight blocks with different response mappings, a small sheet showing the response mapping was placed under the monitor to help them to remember the block-specific instructions. Participants were asked to respond as fast and accurately as possible.

\section{RESULTS}

All analyses[DD2] were performed using R-project software (R Core Team, 2015) and RStudio software (RStudio Team, 2015). Participants had a very high accuracy in all tasks (magnitude classification: mean $=0.97, \mathrm{SD}=0.03$, range $=0.82-1.0$; parity judgement: mean $=0.95, \mathrm{SD}=0.03$, range $=0.87-0.99$; color discrimination: mean $=0.97, \mathrm{SD}=0.03$, range $=$ 0.88-0.997; phoneme detection: mean $=0.94, \mathrm{SD}=0.04$, range $=0.84-0.99$ ). Accuracy data are likely affected by a ceiling effect and thus will not be further analyzed.

Trials with incorrect responses and with reaction times (RTs) shorter than $200 \mathrm{~ms}(\mathrm{~N}=9)$ were excluded from the analyses. For each participant and task combination, trials with RT more than 2.5 SD from the participant's mean were considered outliers and excluded from the analysis (2.9\% of the trials). A preliminary analysis on RTs showed that the order of the tasks was not significant and did not interact with other variables. Therefore, order was excluded from the following analyses. All ANOVAs were Greenhouse-Geisser corrected (Greenhouse \& Geisser, 1959) when the assumption of sphericity was violated; uncorrected degrees of freedom and epsilon values $(\varepsilon G G)$ are reported. Effect sizes are reported following the recommendation of Lakens (2013).

We also calculated the dRT as mean RTs for the right hand minus mean RTs for the left hand, separately for each target number, task, and participant (see Fias et al., 1996; see also Pinhas, Tzelgov, \& Ganor-Stern, 2012; Tzelgov, Zohar-Shai, \& Nuerk, 2013). The dRTs were analyzed by means of a regression model: $\mathrm{dRT} \sim \beta_{0}+\beta_{1} \times$ Number (Fias et al., 1996). The 
slope $\left(\beta_{1}\right)$ was interpreted as a measure of the SNARC effect: larger negative values correspond to a stronger effect.

\subsection{Reaction times}

To investigate the SNARC effect, we first analyzed mean RTs with a repeated measure ANOVA with the within-subject factors task (magnitude, parity, phonology, color), target number $(1,2,3,4,6,7,8,9)$ and response hand (right vs. left). The main effect of number was significant $\left[F(7,217)=21.47, p<0.001, \varepsilon G G=0.70\right.$, generalized eta $\left.{ }^{2}=0.02\right]$. Mean RTs (in ms) were 478 (SD = 52), 477 (57), 501 (53), 501 (56), 501 (58), 493 (52), 485 (53), 493 (49), for the numbers $1,2,3,4,6,7,8,9$, respectively. The main effect of task was significant $[F(3$, $93)=127.67, p<0.001, \varepsilon G G=0.87$, generalized eta $\left.{ }^{2}=0.4\right]$. Mean RTs were $411(S D=45)$, 475 (57), 524 (60), 555 (72), for color, magnitude, parity and phonology tasks, respectively (Figure 1-A). As shown in Table 2, all the comparisons between mean RTs in the different tasks were significant. Moreover, number significantly interacted with task $[F(21,651)=12.30$, $p<0.001, \varepsilon G G=0.43$, generalized eta ${ }^{2}=0.03$ ]. Number and response hand also significantly interacted $\left[F(7,217)=16.03, p<0.001, \varepsilon G G=0.58\right.$, generalized eta $\left.{ }^{2}=0.02\right]$, indicating a SNARC effect. Crucially, the significant three-way interaction number $x$ response hand $x$ task indicates that the SNARC effect varied as a function of task $[F(21,651)=3.01, p=0.01, \varepsilon G G$ $=0.28$, generalized eta $\left.{ }^{2}=0.007\right]$ (Figure 2).

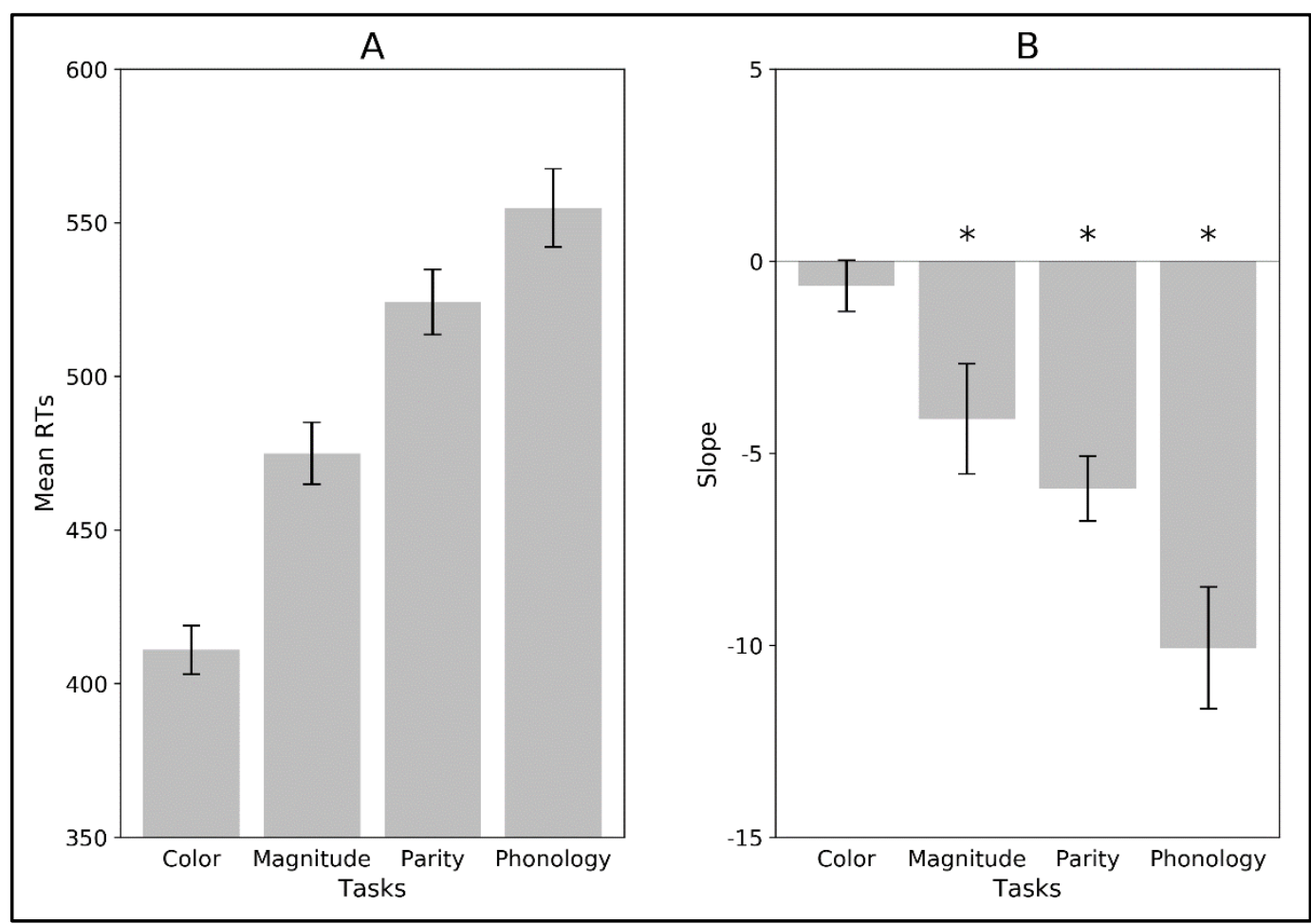

Figure 1. Mean RTs (panel A) and mean slopes (panel B) across the tasks. Magnitude: magnitude classification task; Parity: parity judgement task; Phonology: phoneme detection task; Color: color judgement task. Error bars represent the standard error of the mean. Mean RTs of the tasks are all significantly different from each other (all 
1 ps < 0.001). Except for the difference between magnitude and parity, all the other mean slopes are significantly

2 different from each other (all $p s<0.05$ ). In panel $\mathrm{B}$, asterisks indicate that the mean slope is significantly different

3 from zero (all $p s<0.05$ ).

Table 2. T-tests (one-tailed) comparing the tasks (slope and RTs) against each other and assessing whether the slope distributions were different from zero.

\begin{tabular}{|c|c|c|c|c|c|c|c|}
\hline Tasks & Mean & SD & $t$ & $\mathrm{df}$ & $p$-value & Cohen's dz & Hedges gav \\
\hline \multicolumn{8}{|l|}{ Reaction times } \\
\hline Magnitude vs. Parity & - & - & -8.97 & 31 & $<0.001$ & 1.58 & 0.82 \\
\hline Magnitude vs. Phonology & - & - & -9.86 & 31 & $<0.001$ & 1.74 & 1.19 \\
\hline Magnitude vs. Color & - & - & 8.33 & 31 & $<0.001$ & 1.47 & 1.20 \\
\hline Parity vs. Phonology & - & - & -3.62 & 31 & 0.01 & 0.64 & 0.44 \\
\hline Parity vs. Color & - & - & 14.41 & 31 & $<0.001$ & 2.55 & 2.08 \\
\hline Phonology vs. Color & - & - & 15.41 & 31 & $<0.001$ & 2.72 & 2.33 \\
\hline \multicolumn{8}{|l|}{ Slopes } \\
\hline Magnitude & -4.11 & 8.10 & -2.87 & 31 & 0.018 & 0.51 & 0.70 \\
\hline Parity & -5.92 & 4.79 & -6.98 & 31 & $<0.001$ & 1.23 & 1.70 \\
\hline Phonology & -10.07 & 8.95 & -6.36 & 31 & $<0.001$ & 1.12 & 1.55 \\
\hline Color & -0.64 & 3.75 & -0.97 & 31 & 0.273 & 0.17 & 0.24 \\
\hline Magnitude vs. Parity & - & - & 1.12 & 31 & 0.273 & 0.20 & 0.27 \\
\hline Magnitude vs. Phonology & - & - & 3.75 & 31 & 0.002 & 0.66 & 0.68 \\
\hline Magnitude vs. Color & - & - & -2.47 & 31 & 0.029 & 0.44 & 0.54 \\
\hline Parity vs. Phonology & - & - & 2.59 & 31 & 0.029 & 0.46 & 0.56 \\
\hline Parity vs. Color & - & - & -4.90 & 31 & $<0.001$ & 0.87 & 1.20 \\
\hline Phonology vs. Color & - & - & -5.89 & 31 & $<0.001$ & 1.04 & 1.34 \\
\hline
\end{tabular}

All $p$-values have been corrected with Holm's method. For the calculation of the effect sizes (Cohen's $d_{z}$ and Hedges gav) refers to Lakens (2013).
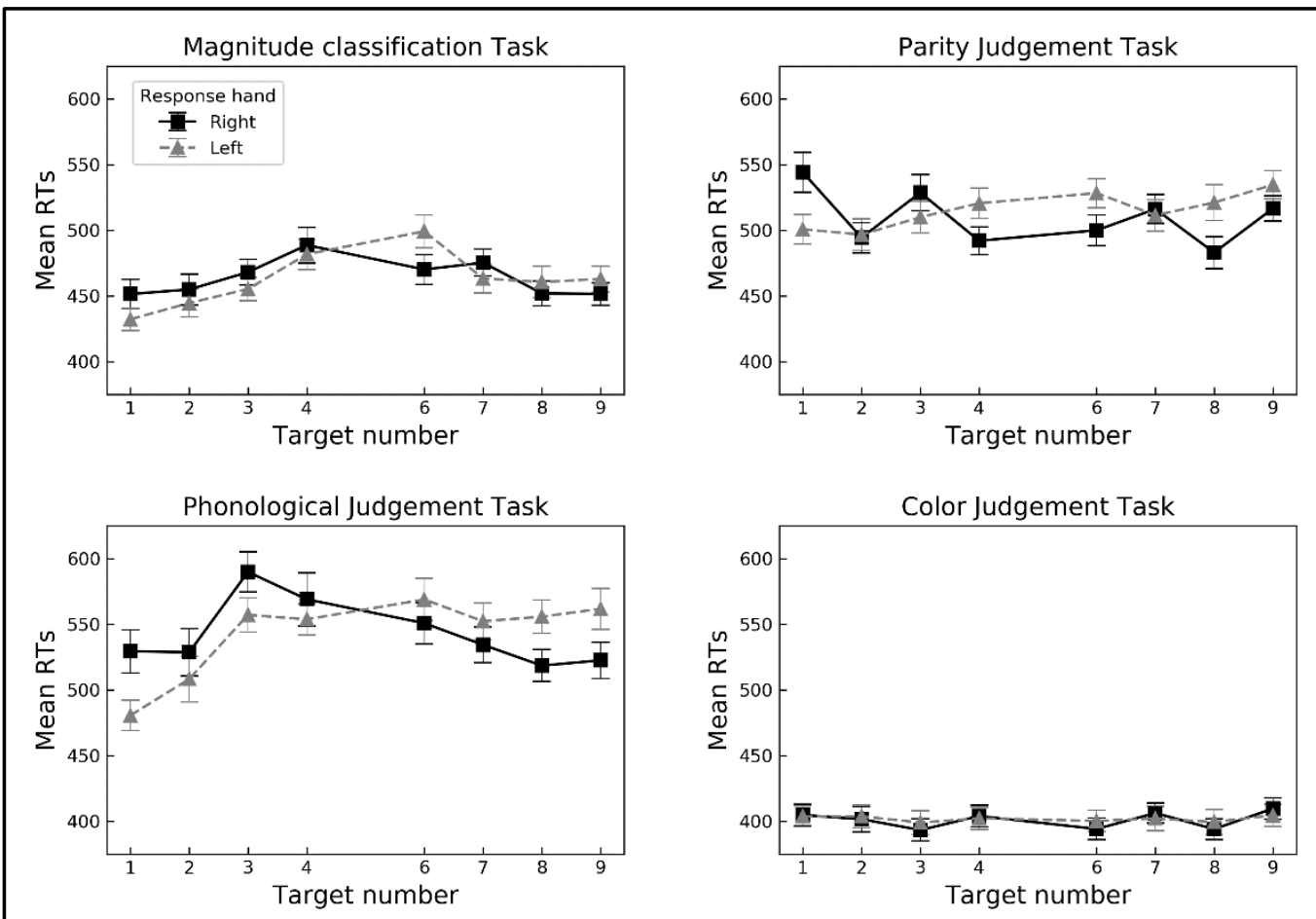

Figure 2. Mean RTs across numbers as a function of response hand (right-hand in black, left-hand in grey), separately for each task. Error bars represent the standard error of the mean. 


\subsection{Slopes}

To further investigate how the SNARC effect varies in the different tasks ${ }^{2}$, the slope ${ }^{3}$ was analyzed with a repeated measures ANOVA with task (magnitude, parity, phonology, color) as within-subject factor. Slope was significantly influenced by task $[F(3,93)=13.78, p<0.001$, $\varepsilon G G=0.87$, generalized eta ${ }^{2}=0.21$ ] (Figure 1-B). Two sets of t-tests were performed to further explore the effect of task on slope (Table 2). First, one-sample t-tests, separately computed for each task, were used to assess whether the slope distributions were significantly less than zero. As shown in Figure 1-B and Table 2, the slopes for the magnitude classification task, the parity judgement task, and the phoneme detection task were significantly different from zero. The slope distribution for the color judgement task was not significantly different from zero, suggesting the absence of a SNARC effect.

Second, two-sample t-tests were used to compare the slope distributions for the different tasks. Except for the magnitude vs. parity comparison, all comparisons were significant (Table 2). These results confirm that there was no SNARC effect for the color judgement task, a medium effect for the magnitude classification and the parity judgement tasks, and the strongest effect for the phoneme detection task. It is worth noting that the pattern of results for the slopes (i.e., the strength of the SNARC effect) mirrors the RT distributions (see panels A and $B$ in Figure 1). The similarity between the results of RTs and of slopes suggests that the SNARC effect is due to the differences in latency rather than to the level of semantic processing. This is also suggested by the unexpected pattern in the slopes: the phoneme detection showed the strongest SNARC effect and the magnitude classification and parity judgement tasks a smaller effect (Figure 1-B). The relationship between the slope distributions of the different tasks were further investigated with Pearson's correlation coefficient (Table 3 and Figure 3). In line with previous studies ( $\rho=0.08$ in Georges, Hoffmann, \& Schiltz, 2017 and $\rho=0.2$ in Georges, Hoffmann, \& Schiltz, 2018), the magnitude classification and the parity judgement tasks did not correlate. The only significant positive correlation emerged between the magnitude classification and phoneme detection tasks. Although this correlation is

\footnotetext{
${ }^{2}$ Split-half reliability was computed for each task. Trials were odd-even half-split based on the order of appearance, separately for each participant, block (response mapping A vs. B) and relevant category (i.e., magnitude classification task: smaller vs. larger than 5; parity judgement task: even vs. odd; phoneme detection task: with vs. without the phonemes; color judgement task: red vs. green). Then, the participant's slope was calculated for each of the two halves. Finally, the Pearson correlation coefficients $\left(r_{S}\right)$ between the slopes of the two halves of each task were calculated and corrected with the Spearman-Brown formula: $r_{S B}=2 r_{S} /\left(1+r_{S}\right)$. The Spearman-Brown corrected correlation coefficients were: $r_{S B}=0.86$ for the magnitude classification task; $r_{S B}$ $=0.83$ for the parity judgement task; $r_{S B}=0.79$ for the phoneme detection task; $r_{S B}=0.58$ for the color judgement task. These coefficients are comparable to or higher than those reported in previous studies (Cipora \& Nuerk, 2013; Georges, Hoffmann \& Schiltz, 2017, 2018). These results indicated excellent reliability for the magnitude classification task, the parity judgement task and the phoneme detection task, and poor reliability for the color judgement task, which may be due to the absence of a SNARC effect for this task.

${ }^{3}$ We also calculated the slopes based on the z-standardized RTs and the same results were found.
} 
consistent with the MNL account, it does not fully support the expected strong correlations between the SNARC effects.

Table 3. Pearson's correlation coefficients between slope distributions of the four tasks (below the diagonal) and between slope and RT distributions (the diagonal).

\begin{tabular}{|c|c|c|c|c|}
\hline & Magnitude & Parity & Phonology & Color \\
\hline Magnitude & $\begin{array}{r}-0.31^{+} \\
(-0.59,0.04)\end{array}$ & (1) & (1) & - \\
\hline Parity & $\begin{array}{r}0.06 \\
(-0.30,0.40)\end{array}$ & $\begin{array}{r}-0.63^{*} \\
(-0.80,-0.36)\end{array}$ & & \\
\hline Phonology & $\begin{array}{r}0.45^{\star} \\
(0.12,0.69)\end{array}$ & $\begin{array}{r}0.24 \\
(-0.11,0.55)\end{array}$ & $\begin{array}{r}-0.36^{*} \\
(-0.63,-0.02)\end{array}$ & \\
\hline Color & $\begin{array}{r}0.27 \\
(-0.09,0.57) \\
\end{array}$ & $\begin{array}{r}-0.002 \\
(-0.35,0.35)\end{array}$ & $\begin{array}{r}0.18 \\
(-0.18,0.50) \\
\end{array}$ & $\begin{array}{r}-0.30^{+} \\
(-0.60,0.06) \\
\end{array}$ \\
\hline
\end{tabular}

The $95 \%$ confidence intervals are presented in parentheses. ${ }^{+} p<0.1,{ }^{*} p<0.05$.

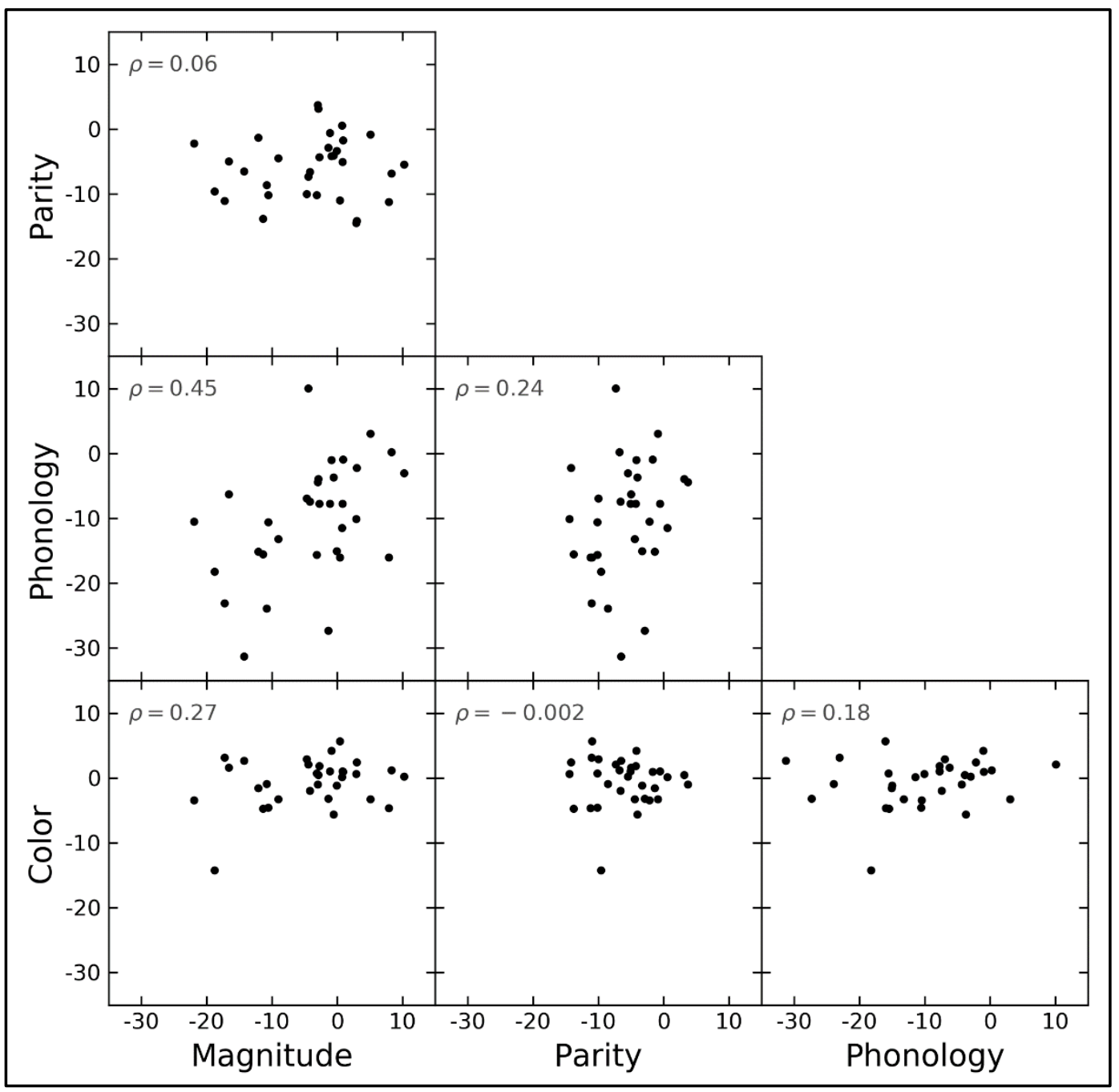

Figure 3. Scatter plots of the correlation between the SNARC effects of the four tasks. Dots represent the participant's mean slope. Magnitude: magnitude classification task; Parity: parity judgement task; Phonology: phoneme detection task; Color: color judgement task. Pearson's correlation coefficients $(\rho)$ are reported in the upper right corner. 


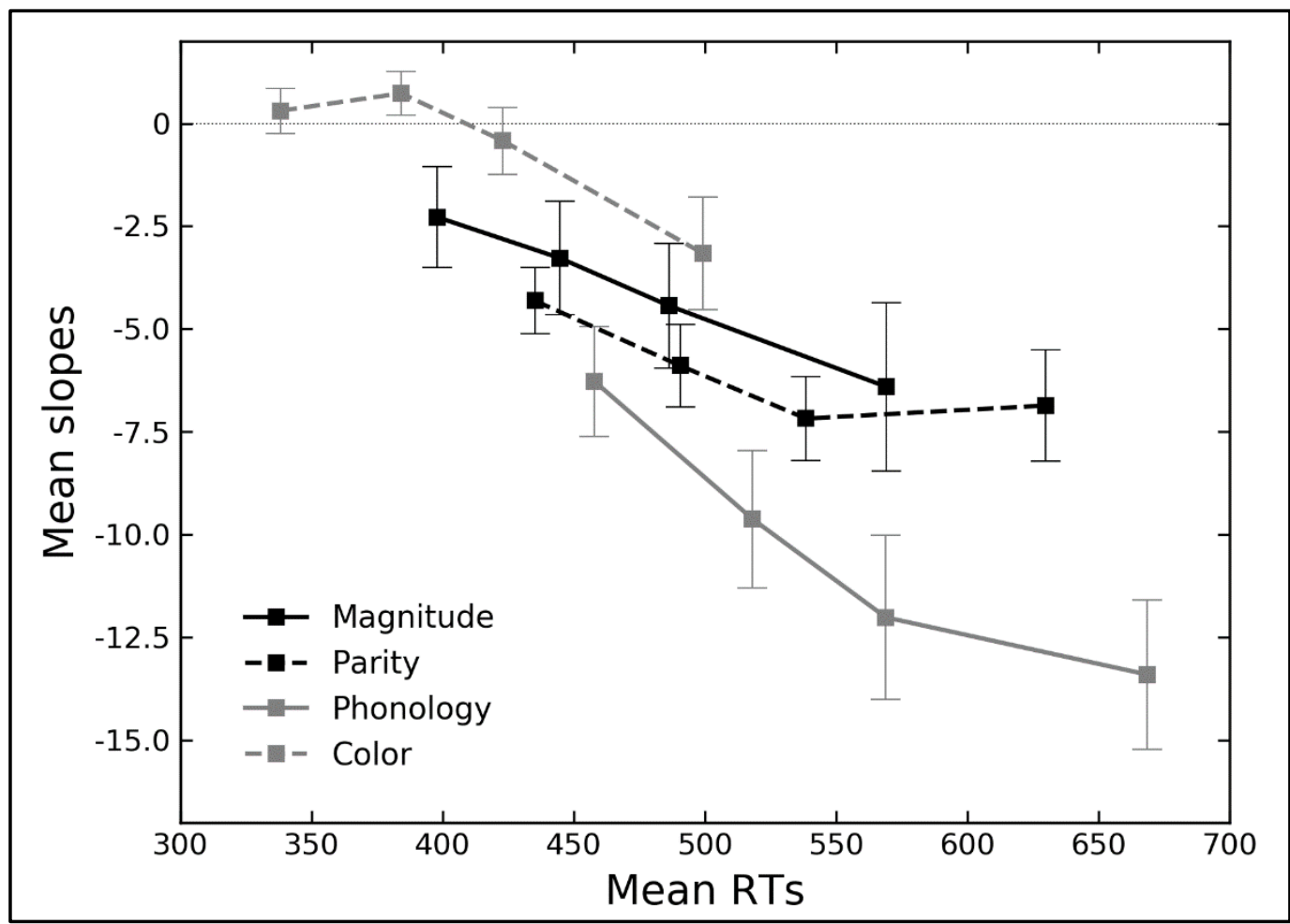

Figure 4. Mean slopes across tasks and bins as a function of RTs. Magnitude: magnitude classification task; Parity: parity judgement task; Phonology: phoneme detection task; Color: color judgement task. For each task, the mean slopes of the four bins are presented: from fastest RTs (leftmost point) to slowest RTs (rightmost point). Error bars represent the standard error of the mean.

The absence of a SNARC effect for the color judgement task may be due to either the very short RTs (i.e., the number processing did not have time to interfere with the response selection) or the fact that a minimum number processing is required in order to produce the SNARC effect and in this task numbers do not reach a sufficient level of processing. To further investigate this possibility, for each participant and task, RTs were rank ordered and divided into 4 bins (Gevers et al., 2006; Ratcliff, 1979). In each bin, slopes were recalculated following the same procedure described above. Figure 4 shows the mean slope distributions across tasks and the bins as a function of RTs and Table 4 reports mean RTs, mean slopes and onesample t-tests assessing whether the slope distributions were significantly less than zero, separately computed for each bin and task. For the parity judgement tasks and the phoneme detection task, a robust SNARC effect emerged in all the bins. The magnitude classification task showed a SNARC effect only in the two slowest bins, a tendency in bin 2 and no effect for the fastest RTs. For the color judgement task, a tendency toward significance emerged only for the bin with longest RTs. All the tasks showed the same patters: mean slope and effect size increased along with response latency. To confirm this pattern, for each task, repeated measure correlation coefficients (Bakdash \& Marusich, 2017, 2018) were calculated between mean slopes and mean RTs. All tasks showed a significant negative correlation between slope and response latency: $r=-0.48(95 \%$ confidence interval $=-0.62,-0.30 ; p<0.001)$ for the 
1 magnitude classification task; $r=-0.32(95 \% \mathrm{Cl}=-0.49,-0.13 ; p<0.01)$ for the parity

2 judgement task; $r=-0.55(95 \% \mathrm{Cl}=-0.68,-0.40 ; p<0.001)$ for the phoneme detection task;

$3 \mathrm{r}=-0.46(95 \% \mathrm{Cl}=-0.61,-0.28 ; p<0.001)$ for the color judgement task. Furthermore, $\mathrm{a}$

4 negative correlation also emerged between the overall mean slopes and overall mean RTs

5 (Table 3 and Figure 5). In fact, both parity judgement and phoneme detection tasks showed

6 a significant negative correlation between overall mean slopes and overall mean RTs, and

7 magnitude classification and color judgement tasks showed a tendency for a negative

8 correlation. These negative correlations further support the idea that the SNARC effect

9 increases along with response latency.

Table 4. Statistics for the four bins and t-tests assessing whether the slopes differed from zero.

\begin{tabular}{|c|c|c|c|c|c|c|c|}
\hline Bins & Mean RTs (SD) & Mean slope (SD) & $t$ & $\mathrm{df}$ & $p$-value & Cohen's dz & Hedges gav \\
\hline \multicolumn{8}{|c|}{ Magnitude } \\
\hline Bin 1 & $398(46)$ & $-2.28(6.95)$ & -1.86 & 31 & $>0.1$ & 0.33 & 0.45 \\
\hline Bin 2 & 445 (53) & $-3.27(7.83)$ & -2.37 & 31 & 0.073 & 0.42 & 0.58 \\
\hline Bin 3 & $486(60)$ & $-4.44(8.59)$ & -2.92 & 31 & 0.022 & 0.52 & 0.71 \\
\hline Bin 4 & $569(77)$ & $-6.41(11.55)$ & -3.14 & 31 & 0.015 & 0.55 & 0.77 \\
\hline \multicolumn{8}{|l|}{ Parity } \\
\hline Bin 1 & $435(47)$ & $-4.32(4.56)$ & -5.35 & 31 & $<0.001$ & 0.95 & 1.31 \\
\hline Bin 2 & 491 (55) & $-5.89(5.65)$ & -5.90 & 31 & $<0.001$ & 1.04 & 1.44 \\
\hline Bin 3 & $538(61)$ & $-7.18(5.74)$ & -7.07 & 31 & $<0.001$ & 1.25 & 1.72 \\
\hline Bin 4 & $630(80)$ & $-6.86(7.69)$ & -5.05 & 31 & $<0.001$ & 0.89 & 1.23 \\
\hline \multicolumn{8}{|c|}{ Phonology } \\
\hline Bin 1 & $458(56)$ & $-6.28(7.59)$ & -4.68 & 31 & $<0.001$ & 0.83 & 1.14 \\
\hline Bin 2 & $518(66)$ & $-9.62(9.45)$ & -5.76 & 31 & $<0.001$ & 1.02 & 1.41 \\
\hline Bin 3 & 569 (76) & $-12.01(11.27)$ & -6.03 & 31 & $<0.001$ & 1.07 & 1.47 \\
\hline Bin 4 & $668(92)$ & $-13.40(10.27)$ & -7.39 & 31 & $<0.001$ & 1.31 & 1.80 \\
\hline \multicolumn{8}{|l|}{ Color } \\
\hline Bin 1 & 338 (37) & $0.31(3.10)$ & 0.56 & 31 & $>0.1$ & 0.10 & 0.14 \\
\hline Bin 2 & $384(40)$ & $0.73(3.04)$ & 1.36 & 31 & $>0.1$ & 0.24 & 0.33 \\
\hline Bin 3 & $423(45)$ & $-0.42(4.59)$ & -0.52 & 31 & $>0.1$ & 0.09 & 0.13 \\
\hline Bin 4 & 499 (61) & $-3.16(7.78)$ & -2.30 & 31 & 0.073 & 0.41 & 0.56 \\
\hline
\end{tabular}




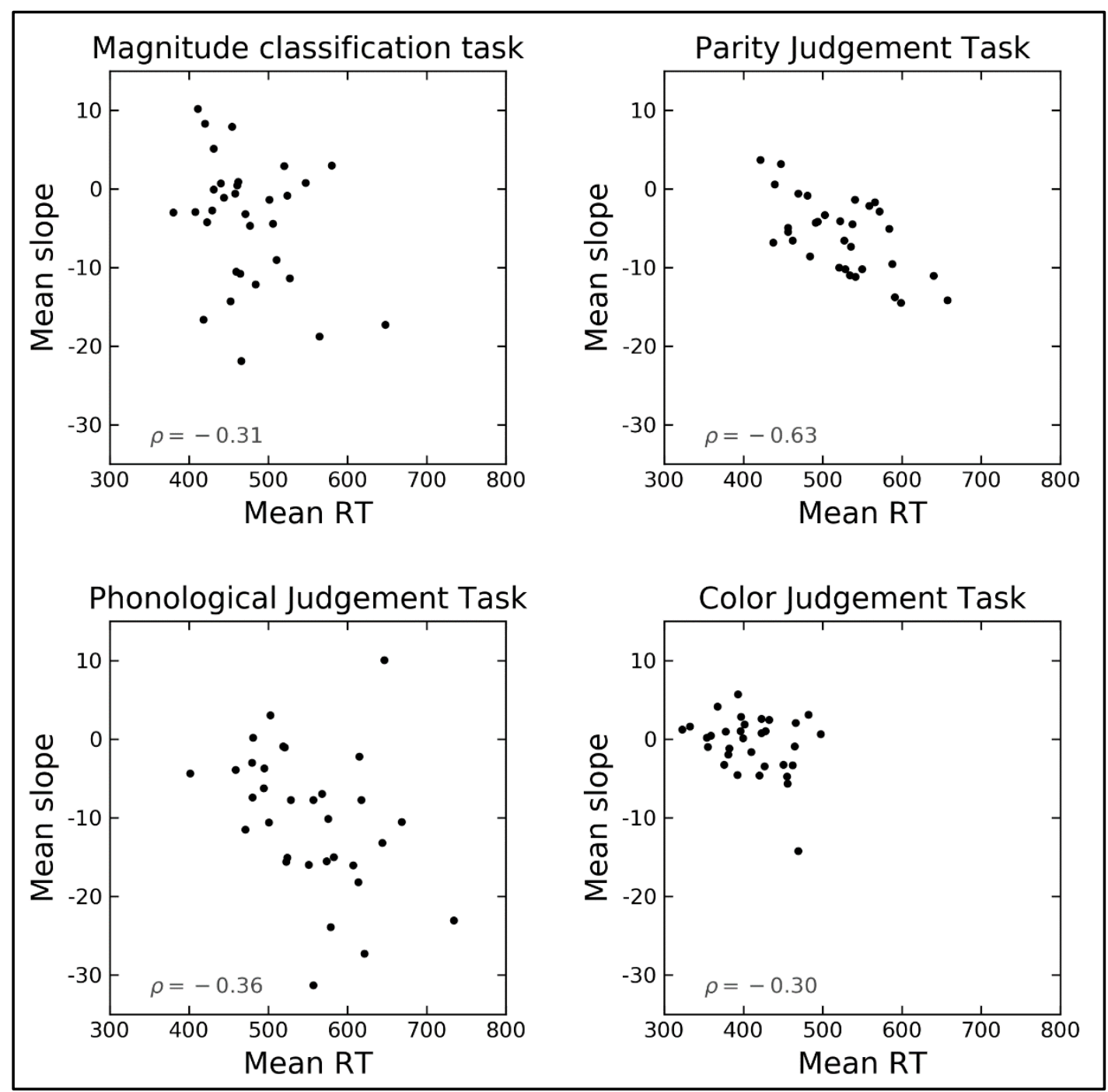

Figure 5. Scatter plots of the correlation between the SNARC effects (mean slope) and response latency (mean RT) in the four tasks. Dots represent the participant's mean slope and RT. Pearson's correlation coefficients $(\rho)$ are reported in the bottom left corner.

\subsection{Shape of the SNARC effect}

According to the dual-route account (Gevers et al., 2006), the SNARC effect should have a categorical shape in the magnitude classification task and a linear shape in the other tasks. To test this prediction, we assessed whether mean $\mathrm{dRT}$ s were best fitted by a categorical predictor or a continuous predictor (for a similar analysis see, for example, Gevers et al., 2006). For each task, we performed a stepwise linear regression analysis (by AIC, with both forward and backward selection method) with mean $\mathrm{dRT}$ as dependent variable and two variables entered in the model as possible predictors: a categorical predictor (a binary variable with the values 1 = "smaller than 5" and 2 = "larger than 5") and a continuous predictor (a continuous variable with the values $1-9$, excluding 5 ). Mean dRTs were calculated aggregating across participants. Table $6 \underline{5}$ reports the models with the variables selected by the analysis. As expected according to the dual-route account, for the magnitude classification task the categorical predictor was 
selected by the analysis, whereas for the parity judgement task and the phoneme detection task the continuous predictor provided the best fit (Figure 6). Due to the lack of a SNARC effect, no predictor was selected for the color judgement task (Figure 6).

Table 45. Results of the stepwise linear regression analysis.

\begin{tabular}{|lrrrrr|}
\hline Selected predictor & $\beta$ & $\mathrm{SE}$ & $\mathrm{t}$ & $p$-value & Adj. $^{2}$ \\
\hline Magnitude classification task & & & & & 0.57 \\
$\quad$ Intercept & 15.14 & 5.23 & 2.90 & 0.028 & \\
Categorical & -23.59 & 7.39 & -3.19 & 0.019 & \\
Parity judgement task & & & & & 0.37 \\
$\quad$ Intercept & 22.61 & 15.02 & 1.51 & 0.183 & \\
Continuous & -5.92 & 2.63 & -2.25 & 0.066 & \\
Phoneme detection task & & & & & 0.91 \\
$\quad$ Intercept & 52.41 & 6.58 & 7.96 & $<0.001$ & \\
$\quad$ Continuous & -10.07 & 1.15 & -8.72 & $<0.001$ & \\
Color judgement task & & & & & - \\
$\quad$ Intercept & -1.73 & 2.00 & -0.87 & 0.415 & \\
\hline
\end{tabular}
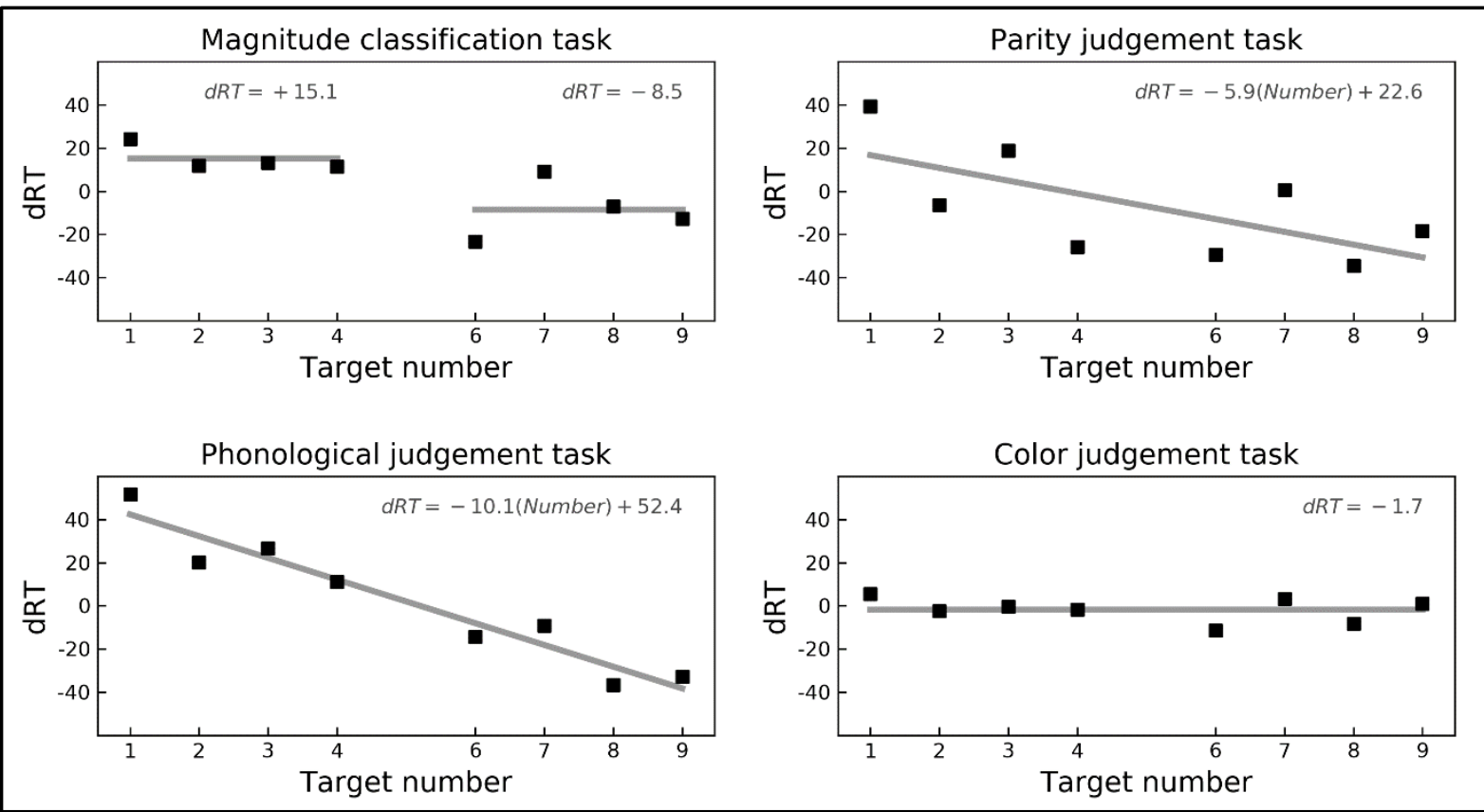

Figure 6. Mean dRTs across numbers, separately for each task. Gray lines represent the model selected in the stepwise analysis: a categorical predictor for the magnitude task; a continuous predictor for the parity and phonology tasks; a model with only intercept for the color task. Above each line, the corresponding model is reported.

\section{DISCUSSION}

The present study aimed to investigate whether the strength of the SNARC effect is influenced by the amount of semantic number processing required by a task. Participants performed two tasks requiring deep semantic number processing (magnitude classification task and parity judgement task) and two tasks requiring to evaluate non-semantic features of 
the numbers (color judgement task and phoneme detection task). Results do not provide evidence for the idea that the level of semantic processing influences the SNARC effect. Conversely, the strength of the SNARC effect was proportional to the overall RTs (the slower the RT, the larger the effect, see Figure 1), suggesting that response latency is the main factor that affects the strength of the SNARC effect. The phoneme detection task had both the longest RTs and the strongest SNARC effect (mean RTs: $555 \mathrm{~ms}$, mean slope: -10.07). The magnitude classification task (mean RTs: $475 \mathrm{~ms}$, mean slope: -4.11) and the parity judgement task (mean RTs: $524 \mathrm{~ms}$, mean slope: -5.92) had faster RTs and showed a weaker SNARC effect of comparable size. The color judgement task had the fastest RTs and showed no evidence of a SNARC effect (mean RTs: $411 \mathrm{~ms}$, mean slope: -0.64). The influence of response latency was also confirmed by the analysis on the rank ordered RTs, which showed that the strength of the SNARC effect was proportional to RTs. In fact, the color judgement task showed a tendency toward significance when the slowest RTs were analyzed (bin 4, mean RTs: $490 \mathrm{~ms}$, mean slope: -3.16) and there was no evidence of a SNARC effect for the fastest RTs of the magnitude classification task (bin 1, mean RTs: $398 \mathrm{~ms}$, mean slope: -2.28). The overall slopes also negatively correlated with overall RTs in the parity judgement and phoneme detection tasks and a tendency emerged in the magnitude classification and color judgement tasks. Although the correlation analyses reported in this paper may be affected by the low sample size $(N=32)$, they are consistent with those reported by Cipora and colleagues (2019), who analyzed the results from a massive sample $(N>1,000)$ in an online assessment of the SNARC effect in a parity judgement task and found a negative correlation between slope and RT. Considering the relationship between the SNARC effects evoked by the four tasks, a positive correlation $(\rho=0.45)$ between slopes emerged only for the magnitude classification and phoneme detection tasks. Finally, the analysis of the shape of the SNARC effect showed that the dRTs of the magnitude classification task were best fit by a categorical predictor, while those of the parity judgement task and phoneme detection task by a continuous predictor.

The MNL account explains the SNARC effect as the result of the association between response locations (left vs. right) and the position of numbers on their spatially oriented representation (small/large numbers on the left/right, respectively) (Dehaene et al., 1993; Hubbard et al., 2005). Therefore, this account predicts that the deeper the access to the MNL, the stronger the influence of the spatial organization of this mental representation should be. Namely, tasks associated with deeper semantic processing (magnitude classification task and parity judgement task) should show a stronger SNARC effect compared to tasks requiring no semantic processing (phoneme detection task and color judgement task). In the latter two tasks, the MNL is thought to be automatically activated (Dehaene et al., 1993; Nuerk, Wood, \& Willmes, 2005) and thus the space-number association should, to some extent, generate a SNARC effect. However, it should be smaller in size compared to tasks that explicitly require 
the processing of semantic aspects of numbers. Our results clearly point in another direction. In fact, not only is the strength of the SNARC effect not proportional to the amount of semantic processing, but also the pattern that emerges (i.e., phoneme detection > parity $\approx$ magnitude $>$ color) cannot be explained by the MNL account. Consistent with a growing amount of evidence showing that the SNARC effect is much more flexible than one would expect under the hypothesis that it is generated from a long-term memory representation (Bächtold et al., 1998; Fias \& van Dijck, 2016; Fias et al., 1996; Georges, Hoffmann, \& Schiltz, 2017, 2018; Shaki \& Fisher, 2008, 2018; van Dijck \& Fias, 2011), our results provide additional empirical evidence that the MNL account cannot adequately explain.

The dual-route model explains the SNARC effect as the result of the competition/cooperation between two parallel information processing streams (Gevers et al., 2006, 2010; Santens \& Gevers, 2008). Since both the unconditional route (i.e., an automatic, long-term space-number mapping) and a conditional route (i.e., an arbitrary task-specific mapping) are always simultaneously activated (Gevers et al., 2006), the SNARC effect should not be affected by the amount of semantic processing required by the task. However, the interference of the unconditional route increases along with response latency, predicting a stronger SNARC effect for longer RTs. Our results provide evidence for this model, showing that the strength of the SNARC effect was influenced by response latency, whereas the amount of semantic processing required by a task had negligible influence. The significant negative correlations between slopes and both overall RTs (Figure 5) and rank ordered RTs (Figure 4) also support the idea that the SNARC effect is mainly influenced by response latency. Our findings are not consistent with the conclusions by Wood and colleagues (2008). In fact, the authors found that "in tasks involving semantic number processing such as parity decision task, the SNARC effect tended to be larger than in non-semantic tasks" (Wood et al., 2008, p. 500). This inconsistency might be due to the fact that we compared the SNARC effect of different tasks in a within-participant design, whereas Wood and colleagues compared different samples. To disentangle the influence of semantic processing and response latency on the SNARC effect, future studies could manipulate the difficulty (and thus RTs) of the task and evaluate whether the SNARC effect increases proportionally to RTs.

According to the MNL account, the SNARC effect originates from a common long-term memory representation regardless of task requirements. Given this common origin, one would expect to observe a strong correlation between the SNARC effects of the different tasks. However, a correlation emerged only between the slopes of the magnitude and phonology tasks (Figure 3). Moreover, the two tasks associated with deep semantic processing (magnitude classification and parity judgement tasks) did not correlate at all $(\rho=0.06)$. The lack of strong correlations is however consistent with the dual-route model and the WM account. The dual-route model assumes that a parallel information processing, which may 
result in competition or cooperation, occurs between the unconditional route and a taskspecific conditional route. Therefore, the interaction between the two routes may vary with different tasks and thus produce uncorrelated SNARC effects. The WM account assumes that the SNARC effect is the result of a temporary binding of numbers to a spatial working memory template. Since the spatial orientation of the number sequence is context- and task-specific, this account is also consistent with uncorrelated SNARC effects. However, it is also possible that the lack of correlation depends on the current sample size and thus this result calls for caution. Future studies with a larger sample size should confirm or reject this result.

The absence of a SNARC effect in the color judgement task might be explained assuming that the decision was made before the automatic activation of the MNL could interfere with the response selection. It is certainly true that this task presents a confound between response latency and amount of semantic processing, inasmuch very fast RTs were combined with a decision process that could be performed without processing the Arabic numerals. Two objections can be made against this argument. First, it is well-established that it is virtually impossible to avoid processing written words or symbols. The color-stroop task is the most prominent example amongst many others. Hence, we do not think that it is valid to assume that the number symbol was not processed at all in the color task. Second, However, this confound is absent in the other tasks. In fact, the phoneme detection task presented the longest response latencies but required no semantic processing; whereas the magnitude classification and parity judgement task had halfway response latencies but required deep semantic processing. Therefore, the MNL account would predict a stronger SNARC effect in the phoneme detection task compared to the color judgement task, because the longer RTs of the former would provide the spatial orientation of the MNL with enough time to interfere with the response selection. However, the highest interference (strongest SNARC effect) is expected in the magnitude task, given that the semantic representation at the origin of the SNARC effect is also the reference frame for the response selection. In fact, the activation of the number on the sematic representation is required to classify the stimulus magnitude. Given the absence of a confound between amount of semantic processing and response latency in the magnitude classification and phoneme detection tasks, the overall results support the idea that the SNARC effect is modulated by response latency rather than semantic processing. However, future studies should further investigate the confound present-introduced byin the color judgement task by for example manipulating the processing of the target number. For example, in a go/no-go color judgement task, where participants are asked to discriminate the color only for a specific subset of the number range (e.g., go trials: 1, 4, 6, 9).

We interpreted our results as evidence in favor of the dual-route model. However, is there an alternative interpretation that would allow to explain these results within the MNL framework? In principle the answer is yes. In fact, along with the idea that the deeper the 
semantic processing required by a task, the stronger the in interference from the spatial orientation of the MNL is $\frac{1}{1}$ one could also assume that this interference increases proportionally to response latency. Similar to what follows fromproposed for the dual-route model, the longer the decision process takes, the higher the chance for the spatial orientation of the MNL to interfere with the response selection is. Although this seems a plausible explanation, we believe that our results do not support this interpretation. The MNL account and the dual-route model make divergent predictions for the relationship between the SNARC effect and the rank ordered RTs in the bin analysis. If one assumes that the interference of the semantic representation increases with the duration of the decision process, the MNL account predicts that the strength of the SNARC effect increases proportionally to the RTs in the parity judgement, phoneme detection and color judgement tasks. This is indeed consistent to what we observed in the bin analysis (see Figure 4 and Table 4). On the contrary, for the magnitude classification task, the strength of the SNARC effect should remain constant and not be affected by response latency. In fact, in this task, the semantic representation must be explicitly activated, since it is strictly required to classify the magnitude of the number, and thus its activation level should not be modulated by response latency. However, this prediction is inconsistent with the results of the magnitude classification task: no SNARC effect in bin 1, a tendency in bin 2, and a SNARC effect emerging only in bin 3 and 4 (see Figure 4 and Table 4). At this point, it is worth drawing attention to a fundamental difference between the two accounts. Contrary to the MNL account, increasing strength of the SNARC effect with longer response latencies in the magnitude task is an important feature of the dual-route model and explains the shape of the SNARC effect in this task (see Introduction and next paragraph). To sum up, even an alternative interpretation of the MNL account cannot explain the observed relationship between the SNARC effect and the rank ordered RTs.

Further evidence in favor of the dual-route model is also provided by the analysis of the shape of the SNARC effect. Consistent with the prediction of this model (Gevers et al., 2006), we found a categorical shape for the SNARC effect in the magnitude classification task and a linear shape for the other tasks. This result is consistent with the meta-analysis of Wood and colleagues (2008), who found that in $60 \%$ of the studies included in their review a categorical predictor alone could account for the SNARC effect in the magnitude comparison task. The categorical shape of the SNARC effect in the magnitude classification task also provides further evidence for the idea that response latency has a major impact on this effect. In fact, the categorical shape depends on the fact that the processing of the numbers closer to the fixed standard (i.e., 5) is more time consuming due to the distance effect (Gevers et al., 2006; see also Moyer \& Landauer, 1967; Verguts, Fias, \& Stevens, 2005). This longer processing provides more time to the unconditional route to interfere with the decision process, and thus generates a stronger SNARC effect for the numbers closer to the fixed standard. 
The WM account assumes that the SNARC effect emerges by the positioning of numbers within a temporary spatial template loaded in working memory (Abrahamse, van Dijck, \& Fias, 2016; Fias \& van Dijck, 2016; van Dijck \& Fias, 2011; van Dijck, Gevers, \& Fias, 2009; van Dijck et al., 2012, 2014). The space-number association results from the fact that this template is spatially oriented from left to right (small to large numbers). Since the space-number association is not an intrinsic property of the number representation but it is instead temporary temporarily created in working memory (Abrahamse, van Dijck, \& Fias, 2016), this account also does not predict that deeper semantic processing generates a stronger SNARC effect. Although this account does not specify whether and how response latency affects the strength of the SNARC effect, it is not incompatible with the results reported in the current study. However, it should be specified which mechanism is responsible for the influence of response latency on the strength of the SNARC effect. The WM account can also explain the categorical shape of the SNARC effect by assuming that the spatial template temporarily loaded in working memory is subject to the distance effect. However, it is worth mentioning that, according to the WM account, the distance effect is generated in working memory, whereas for the dual-route model this effect results from a feature of the long-term memory representation of the numbers (Abrahamse, van Dijck, \& Fias, 2016).

Despite the very low error rate, the RTs of the phoneme detection task were significantly longer than those of the other tasks. This task required to judge the presence or absence of a group of three phonemes in the number words and thus to transcode the stimulus between two symbolic notations: Arabic to phonological (Nuerk, Moeller, \& Willmes, 2015; Silke Göbel et al., 2014). Namely, the visually presented Arabic number had to be transcoded into the internal phonological representation, on which then the search for a specific phoneme could be performed. The phoneme detection task was therefore more demanding compared to the other three tasks and this could explain why this task had the longest RTs and thus the strongest SNARC effect.

The tasks investigated in the current study required explicit spatial-directional processing (i.e., response keys were lateralized) and explicit (magnitude classification task) or implicit (parity judgement task, color judgement task, and phoneme detection task) magnitude processing (for a more detailed description of this task classification see Shaki \& Fischer, 2018). In a recent study, Shaki and Fischer (2018) provided evidence that to some extent the SNARC effect might be an artifact of the procedure used to measure it. In fact, using magnitude classification and parity judgement tasks with go/no-go responses, Shaki and Fischer showed that the horizontal SNARC SNAS requires explicit activation of spatial representation (in stimulus or response code) or explicit magnitude processing. On the other hand,-a vertical SNARCS_effect emerged also with implicit spatial and magnitude processing. Consistent with our results, Shaki and Fischer's findings are also difficult to reconcile with the assumption of a 
1 left-to-right horizontally organized long-term memory representation of numbers. However, the 2 results reported in the current manuscript might be limited to tasks with left/right horizontally 3 distributed response keys. Future studies should investigate whether our results are limited 4 only to tasks that require an explicit spatial-directional processing or whether they are also 5 valid for tasks with implicit spatial processing (e.g., go/no-go responses).

6 In sum, the results reported in the present study suggest that the strength of the SNARC 7 effect is mainly modulated by overall response latencies rather than by the amount of semantic 8 processing required by the tasks. These results are in line with the prediction of the dual-route 9 model and can also be explained within the framework of the WM account. On the other hand, 10 our data provide evidence against the idea that deeper semantic processing generates a 11 stronger SNARC effect, as it would be expected according to the MNL account. 
1 Acknowledgement[DD3]

2 This work was supported by a grant (DI 2361/1-1) from Deutsche Forschungsgemeinschaft 3 (DFG, German Research Council) to Daniele Didino.

4

5

6 


\section{REFERENCES}

Abrahamse, E., van Dijck, J.-P., \& Fias, W. (2016). How Does Working Memory Enable Number-Induced Spatial Biases? Frontiers in Psychology, 7, 977.

Bächtold, D., Baumüller, M., \& Brugger, P. (1998). Stimulus-response compatibility in representational space. Neuropsychologia, 36(8), 731-735

Bakdash, J.Z., \& Marusich, L.R. (2017). Repeated Measures Correlation. Frontiers in Psychology, 8, 456. doi: 10.3389/fpsyg.2017.00456

Bakdash, J.Z., \& Marusich, L.R. (2018). rmcorr: Repeated Measures Correlation. R package version 0.3.0. https://CRAN.R-project.org/package=rmcorr

Brainard, D. H. (1997). The Psychophysics Toolbox. Spatial vision, 10(4), 443-446.

Cipora, K., \& Nuerk, H. C. (2013). Is the SNARC effect related to the level of mathematics? No systematic relationship observed despite more power, more repetitions, and more direct assessment of arithmetic skill. The Quarterly Journal of Experimental Psychology, 66(10), 1974-1991. doi: 10.1080/17470218.2013.772215

Cipora, K., Soltanlou, M., Reips, U.-D., Nuerk, H.-C. (2019). The SNARC and MARC effects measured online: Large-scale assessment methods in flexible cognitive effects. Behavior Research Methods. doi: 10.3758/s13428-019-01213-5

Cipora, K., \& Wood, G. (2017). Finding the SNARC instead of hunting it: A $20 \star 20$ monte carlo investigation. Frontiers in Psychology, 8, 1194.

Dehaene, S., Bossini, S., \& Giraux, P. (1993). The mental representation of parity and number magnitude. Journal of Experimental Psychology: General, 122(3), 371-396.

Dehaene, S., Naccache, L., Le Clec'H, G., Koechlin, E., Mueller, M., Dehaene-Lambertz, G., van de Moortele, P.-F., \& Le Bihan, D. (1998). Imaging unconscious semantic priming. Nature, 395(6702), 597-600. doi: 10.1038/26967

Fias, W. (2001). Two routes for the processing of verbal numbers: Evidence from the SNARC effect. Psychological Research, 65, 250-259.

Fias, W., Brysbaert, M., Geypens, F., \& D'Ydewalle, G. (1996). The importance of magnitude information in numerical processing: Evidence from the SNARC effect. Mathematical Cognition, 2(1), 95-110.

Fias, W., \& van Dijck, J.-P. (2016). The temporary nature of number-space interactions. Canadian Journal of Experimental Psychology, 70(1), 33-40.

Fischer, M. H., \& Shaki, S. (2014). Spatial associations in numerical cognition - From single digits to arithmetic. Quarterly Journal of Experimental Psychology, 67(8), 1461-1483.

Georges, C., Hoffmann, D., \& Schiltz, C. (2017). How and why do number-space associations co-vary in implicit and explicit magnitude processing tasks? Journal of Numerical Cognition, 3(2), 182-211. 
Georges, C., Hoffmann, D., \& Schiltz, C. (2018). Implicit and explicit number-space associations differentially relate to interference control in young adults with ADHD. Frontiers in Psychology, 9, 775.

Gevers, W., Santens, S., Dhooge, E., Chen, Q., Van den Bossche, L., Fias, W., \& Verguts, T. (2010). Verbal-spatial and visuospatial coding of number-space interactions. Journal of Experimental Psychology: General, 139(1), 180-90.

Gevers, W., Verguts, T., Reynvoet, B., Caessens, B., \& Fias, W. (2006). Numbers and space: A computational model of the SNARC effect. Journal of Experimental Psychology: Human Perception and Performance, 32(1), 32-44.

Ginsburg, V., and Gevers, W. (2015). Spatial coding of ordinal information in short-and longterm memory. Frontiers in Human Neuroscience. 9:8. doi: 10.3389/fnhum.2015.00008

Göbel, S. M. (2015). Up or down? Reading direction influences vertical counting direction in the horizontal plane-a cross-cultural comparison. Frontiers in psychology, 6, 228.

Göbel, S. M., Maier, C. A., \& Shaki, S. (2015). Which numbers do you have in mind? Number generation is influenced by reading direction. Cognitive processing, 16(1), 241-244.

Göbel, S. M., Shaki, S., \& Fischer, M. H. (2011). The cultural number line: a review of cultural and linguistic influences on the development of number processing. Journal of CrossCultural Psychology, 42(4), 543-565.

Göbel, S. M., Watson, S. E., Lervåg, A., \& Hulme, C. (2014). Children's arithmetic development: It is number knowledge, not the approximate number sense, that counts. Psychological science, 25(3), 789-798.

Greenhouse, S. W., and Geisser, S. (1959). On methods in the analysis of profile data. Psychometrika, 24, 95-112.

Huber, S., Klein, E., Moeller, K., \& Willmes, K. (2016). Spatial-Numerical and ordinal positional associations coexist in parallel. Frontiers in Psychology, 7, 438. doi:

\subsection{9/fpsyg.2016.00438}

Nuerk, H.-C., Moeller, K., \& Willmes, K. (2015). Multi-digit Number Processing: Overview, Conceptual Clarifications, and Language Influences. In Cohen Kadosh, R., \& Dowker, A. (Eds.), Oxford library of psychology. The Oxford handbook of numerical cognition, New York, NY, US: Oxford University Press, pp. 106-139.

Hubbard, E. M., Piazza, M., Pinel, P., and Dehaene, S. (2005). Interactions between number and space in parietal cortex. Nature Reviews Neuroscience, 6(6), 435-448.

Koechlin, E., Nacchache, L., Block, E., \& Dehaene, S. (1999). Primed numbers: exploring the modularity of numerical representations with masked and unmasked semantic priming. Journal of Experimental Psychology: Human Perception and Performance, 25(6), 18821905. doi: 10.1037/0096-1523.25.6.1882 
Kunde, W., Kiesel, A., \& Hoffmann, J. (2003). Conscious control over the content of unconscious cognition. Cognition, 88(2), 223-242. doi:10.1016/S0010-0277(03)00023-4

Lakens, D. (2013). Calculating and reporting effect sizes to facilitate cumulative science: a practical primer for t-tests and ANOVAs. Frontiers in psychology, 4, 863.

Maxfield, L. (1997). Attention and semantic priming: A review of prime task effects. Consciousness and cognition, 6(2-3), 204-218. doi: 10.1006/ccog.1997.0311

Moyer, R. S., \& Landauer, T. K. (1967). Time required for judgements of numerical inequality. Nature, 215(5109), 1519-1520.

Naccache, L., \& Dehaene, S. (2001). Unconscious semantic priming extends to novel unseen stimuli. Cognition, 80(3), 223-237. doi: 10.1016/S0010-0277(00)00139-6

Nuerk, H.-C., Wood, G., \& Willmes, K. (2005). The universal SNARC effect: The association between number magnitude and space is amodal. Experimental Psychology, 52(3), 187194.

Pelli, D. G. (1997). The VideoToolbox software for visual psychophysics: Transforming numbers into movies. Spatial vision, 10(4), 437-442.

Pinhas, M., Tzelgov, J., \& Ganor-Stern, D. (2012). Estimating linear effects in ANOVA designs: the easy way. Behavior research methods, 44(3), 788-794.

Proctor, R. W., \& Cho, Y. S. (2006). Polarity correspondence: A general principle for performance of speeded binary classification tasks. Psychological Bulletin, 132(3), 416442.

R Core Team (2015). R: A language and environment for statistical computing. Vienna: $\mathrm{R}$ Foundation for Statistical Computing.

Ratcliff, R. (1979). Group reaction time distributions and an analysis of distribution statistics. Psychologican Bulletin, 86(3), 446-461.

RStudio Team (2015). RStudio: Integrated development for R. Boston, MA: RStudio, Inc.

Santens, S., \& Gevers, W. (2008). The SNARC effect does not imply a mental number line. Cognition, 108(1), 263-270.

Schroeder, P. A., Nuerk, H. C., \& Plewnia, C. (2017a). Prefrontal neuromodulation reverses spatial associations of non-numerical sequences, but not numbers. Biological Psychology, 128, 39-49. doi: 10.1016/j.biopsycho.2017.07.008

Schroeder, P. A., Nuerk, H. C., \& Plewnia, C. (2017b). Switching between multiple codes of SNARC-like associations: Two conceptual replication attempts with anodal tDCS in shamcontrolled cross-over design. Frontiers in Neuroscience, 11, 654. doi: 10.3389/fnins.2017.00654

Schroeder, P. A., Nuerk, H. C., \& Plewnia, C. (2017c). Space in numerical and ordinal information: A common construct? Journal of Numerical Cognition, 3(2), 164-181. doi: 10.5964/jnc.v3i2.40 
Shaki, S., Fischer, M. H., \& Göbel, S. M. (2012). Direction counts: A comparative study of spatially directional counting biases in cultures with different reading directions. Journal of Experimental Child Psychology, 112(2), 275-281.

Shaki, S., \& Fischer, M. H. (2008). Reading space into numbers: A cross-linguistic comparison of the SNARC effect. Cognition, 108(2), 590-599.

Shaki, S., \& Fischer, M. H. (2018). Deconstructing spatial-numerical associations. Cognition, $175,109-113$.

Shaki, S., Fischer, M. H., \& Petrusic, W. M. (2009). Reading habits for both words and numbers contribute to the SNARC effect. Psychonomic Bulletin \& Review, 16(2), 328-331.

Tzelgov, J., Zohar-Shai, B., \& Nuerk, H. C. (2013). On defining quantifying and measuring the SNARC effect. Frontiers in psychology, 4, 302.

van Dijck, J.-P., Abrahamse, E. L., Acar, F., Ketels, B., \& Fias, W. (2014). A Working Memory Account of the Interaction between Numbers and Spatial Attention. Quarterly Journal of Experimental Psychology, 67(8), 1500-1513.

van Dijck, J.-P., \& Fias, W. (2011). A working memory account for spatial-numerical associations. Cognition, 119(1), 114-119.

van Dijck, J.-P., Gevers, W., \& Fias, W. (2009). Numbers are associated with different types of spatial information depending on the task. Cognition, 113(2), 248-253.

van Dijck, J.-P., Gevers, W., Lafosse, C., \& Fias, W. (2012). The Heterogeneous Nature of Number-Space Interactions. Frontiers in Human Neuroscience, 5, 182.

Van Dijck, J.-P., Ginsburg, V., Girelli, L., \& Gevers, W. (2015). Linking numbers to space: From the mental number line towards a hybrid account. In Cohen Kadosh, R., \& Dowker, A. (Eds.), Oxford library of psychology. The Oxford handbook of numerical cognition, New York, NY, US: Oxford University Press, pp. 89-105.

Verguts, T., Fias, W., \& Stevens, M. (2005). A model of exact small-number representation. Psychonomic Bulletin \& Review, 12(1), 66-80.

Wood, G., Willmes, K., Nuerk, H.-C., \& Fischer, M. H. (2008). On the cognitive link between space and number: a meta-analysis of the SNARC effect. Psychology Science Quarterly, 50(4), 489-525. 